JOURNAL OF MODERN OPTICS, 2001, VOL. 48, NO. 7, 1247-1253

\title{
Optical propagation of fractal fields. Experimental analysis in a single display
}

\author{
OSVALDO TRABOCCHI, SERGIO GRANIERI and WALTER \\ D. FURLAN* \\ Centro de Investigaciones Ópticas (CIOp), C.C. 124, (1900) La Plata, \\ Argentina \\ * Departamento de Óptica, Universitat de València, \\ E-46100 Burjassot, Spain.
}

(Received 3 October 2000; revision received 13 December 2000)

\begin{abstract}
An experimental device to show in a single display all the diffraction patterns generated by a $1 \mathrm{D}$ fractal structure is proposed. It is found that in addition to being the optimum display to see the evolution of the diffracted field through free space, some interesting features, such as continuous evaluation of self-similarity from the object to the far field, can be obtained experimentally.
\end{abstract}

\section{Introduction}

Many natural phenomena and physical structures, such as phase transition, turbulence, or optical textures, can be analysed and described using the fractal approach [1]. For this reason, the properties of diffraction patterns produced by fractal objects and their potential applications have attracted the attention of several researchers during recent years. Most research has been devoted to the study of diffraction properties of fractals in the Fraunhofer region [2, 3] but there are only a few studies concerning Fresnel diffraction. In [4] it was numerically demonstrated that during Fresnel diffraction of a Cantor set, the irradiance distributions along the optical axis have a periodicity that depends on the level of the set. In the same reference it was also shown that the intensity distributions at transverse planes show a partial self-similar behaviour which is increased when moving towards the Fraunhofer region. In [5], evolution of the complex amplitude of a Cantor fractal propagating through free space was also numerically evaluated and represented in a two-dimensional display where one axis is the transversal coordinate and the other is a certain bounded function of the axial coordinate. This kind of two-dimensional representation has been shown very useful since it contains most of the relevant information about the diffraction patterns of such objects. However, to our knowledge there is no experimental setup designed to obtain them. In this work an optical arrangement is proposed to visualize all the diffraction patterns generated by a fractal structure simultaneously in a single display. Additionally it is shown that some interesting features of fractals, such as evolution of self-similarity under propagation, can be obtained from this experimental result. Comparisons between numerical and experimental results show the good performance of the proposed arrangement. 


\section{2D finite display of $1 \mathrm{D}$ diffractals}

Consider the irradiance distribution of the light diffracted (according to the Fresnel-Kirchhoff approximation) by a 1D fractal object represented by an amplitude transmittance function $t\left(x_{0}\right)$. When it is illuminated by a monochromatic plane wave of wavelength $\lambda$, this irradiance pattern over a transverse plane at a distance $R_{0}$, from the object is given by

$$
I\left(x ; R_{0}\right)=\frac{1}{\left(\lambda R_{0}\right)^{2}}\left|\int_{-\infty}^{+\infty} t\left(x_{0}\right) \exp \left\{\frac{\mathrm{i} \pi x_{0}^{2}}{\lambda R_{0}}\right\} \exp \left\{\frac{-\mathrm{i} 2 \pi x x_{0}}{\lambda R_{0}}\right\} \mathrm{d} x_{0}\right|^{2} .
$$

It is well known that if the parallel illumination is replaced by a convergent cylindrical wavefront, the diffraction patterns previously obtained for values from $R_{0}=0$ to $R_{0}=\infty$ are now confined in a spatial volume axially limited by the object plane and by the plane of the lens focal line [6]. If the distance from the focal plane to the object is $z$, the diffraction pattern defined by $R_{0}$ in equation (1) is now obtained at a distance $R$ given by (see figure $1(a)$ ).

$$
R=\frac{z R_{0}}{z-R_{0}},
$$

but affected by a scale factor $M$ that can be expressed as

$$
M=\frac{R}{R_{0}}=\frac{z}{z-R_{0}} .
$$

In this way, by use of convergent illumination the irradiance distribution obtained at a finite distance $R$ from the object can be expressed, except for a multiplicative factor, in terms of the corresponding one with parallel illumination, as:

$$
I^{\prime}(x ; R, z)=I\left(\frac{x}{M} ; R_{0}\right) .
$$

Due to the 1D nature of the input function each individual diffraction pattern presents no variations along the vertical axis $y$ (see figure 1(a)). As we are looking for a $2 \mathrm{D}$ single display in which profiles of these patterns are laid out side by side, a way is needed to select strips of these patterns and to superimpose them in a single plane. To do this, consider the optical arrangement sketched in figure 1(b). 1D horizontal strips of infinitesimal width can be defined in the object, each one at a different height $y_{R_{0}}$ to be used as a single diffraction-order channel. By use of a varifocal lens ( $L$ in figure 1(b)) whose power varies along the vertical axis, each one of the axially distributed diffraction patterns can be imaged independently at a different height at the output plane. The system works in parallel, so in the final image all 1D diffraction patterns of the input are obtained simultaneously. As mentioned earlier, the focal length, $f$, of the lens $L$, must be different for each channel, in other words it must be a continuous function of the coordinate $y$, as is deduced as follows. For each diffraction pattern $R_{0}$ located at a variable distance $(l-R)$ from $L$, we select the channel centred at $y_{R_{0}}$ in such a way that the varifocal lens images this pattern at the output plane at a fixed distance $a^{\prime}$ (see figure $2(b)$ ). The power of such a lens for each channel can be straightforwardly obtained from the Gaussian lens equation as: 

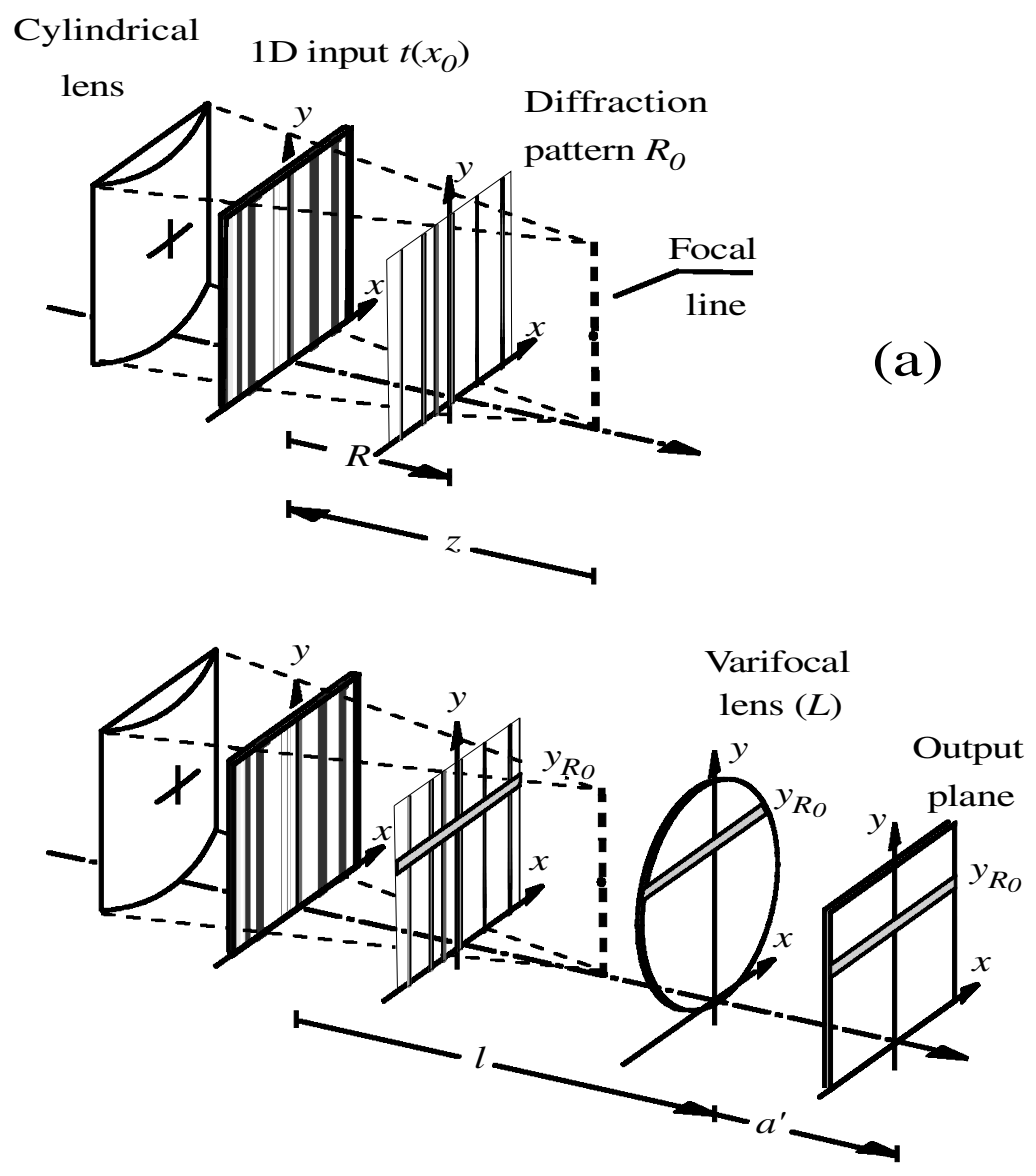

(b)

Figure 1. (a) Diffraction patterns of a $1 \mathrm{D}$ object generated by a convergent cylindrical wavefront. The pattern $R_{0}$ in equation (1) is obtained at a distance $R$ from the input.

(b) Setup for the simultaneous visualization of all Fresnel diffraction patterns of $1 \mathrm{D}$ fractals. The varifocal lens images all the individual patterns between the input and the focal line in a single output plane.

$$
\varphi(R)=f^{-1}(R)=\frac{l-R+a^{\prime}}{(l-R) a^{\prime}} .
$$

The power lens variation with transverse displacement parallel to the line focus $\varphi(y)$ is arbitrary, and then it can be matched with the almost linear variation of an ophthalmic varifocal lens. In this class of lens there is a continuous linear transition between two optical powers $\varphi_{0}$ and $\varphi_{h}$ corresponding to the so-called near portion and distance portion respectively [7]; i.e.:

$$
\varphi(y)=\frac{\left(\varphi_{h}-\varphi_{0}\right)}{h} y+\varphi_{0}
$$

where $h$ is the extent of the so-called progression zone. This use of the varifocal lens, besides being inexpensive, provides an output in which the diffraction patterns are 
ordered from the near field to the far field or vice versa. The boundary conditions can be obtained by imposing that for $y=0$ the output displays the image of the pattern corresponding to $R_{0}=R=0$ (image of the object $t\left(x_{0}\right)$ ), while for $y=h$ the output displays the image of the pattern corresponding to $R_{0} \rightarrow \infty$, i.e. $R=-z$ (image of the object Fourier transform). Any other particular diffraction pattern characterized by the parameter $R_{0}$ in equation (1) is obtained (as can be deduced from equations (2) (5), and (6)) in the horizontal strip of the output located at the coordinate $y_{R_{0}}$ satisfying:

$$
y_{R_{0}}=\frac{h}{1+\alpha / R_{0}},
$$

where $\alpha$ is defined in terms of the system parameters as $\alpha=\left(\varphi_{h}-\varphi_{0}\right) l^{2}$. On the other hand, the scale factor, $M_{R_{0}}$, of each diffraction pattern at the output plane, is obtained by the product of the magnification resulting from the use of cylindrical illumination $M$ of equation (2), and the lateral magnification provided by the varifocal lens:

$$
M_{L}(R)=\frac{-a^{\prime}}{l-R}
$$

Thus, using equation (2), we obtain

$$
M_{R_{0}}=M M_{R_{0}}=m_{0}\left(1+R_{0} / \alpha\right),
$$

where $m_{0}$ is the magnification of the image of the pattern corresponding to $R_{0}=0$. Summarizing, equations (7) and (9) represent the localization and magnification of a single diffraction pattern of the fractal object at the output $2 \mathrm{D}$ display.

In the next section some experimental results obtained with this proposal are presented.

\section{Experimental results}

The system of figure $1(b)$ was assembled with an ophthalmic varifocal lens of powers $\varphi_{0}=+2.75 \mathrm{D}$ and $\varphi_{h}=+5.75 \mathrm{D}$ and the following values for distances: $z=426 \mathrm{~mm}, l=646 \mathrm{~mm}$ and $a^{\prime}=831 \mathrm{~mm}$. Figure $2(a)$ illustrates the experimental results registered by a CCD camera using a tetraedic Cantor set grating as input object. For comparison the same result was obtained numerically and is shown in figure $2(b)$. The use of a varifocal lens in the experiments produces $y$ deflections contaminating the separation of images corresponding to different channels. However, this effect has a negligible impact on the final image because the cylindrical illuminating wavefront imposes a quasi-1D behaviour on the diffracted field for each channel.

The experimental result shown in figure $2(a)$ can also be favourably compared with the numerical result reported in [5]. It can be seen that, unlike the previously reported representation, in which the transverse coordinate is plotted against the logarithm of the axial distance, in our display the $x$ coordinate is plotted against a hyperbolic function of the axial distance (see equation (7)). This representation provides a fine sampling of the diffracted field near the object, precisely where the diffraction patterns change rapidly, and a coarse sampling in the far field where the variation of the diffraction patterns with $R_{0}$ is low. 
(a)

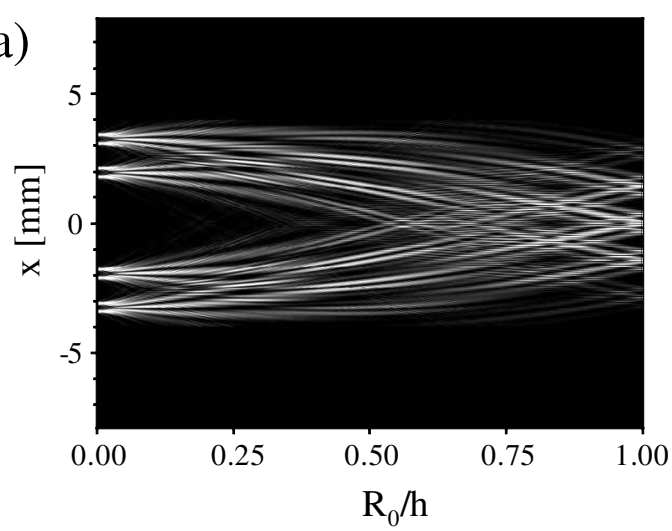

(b)

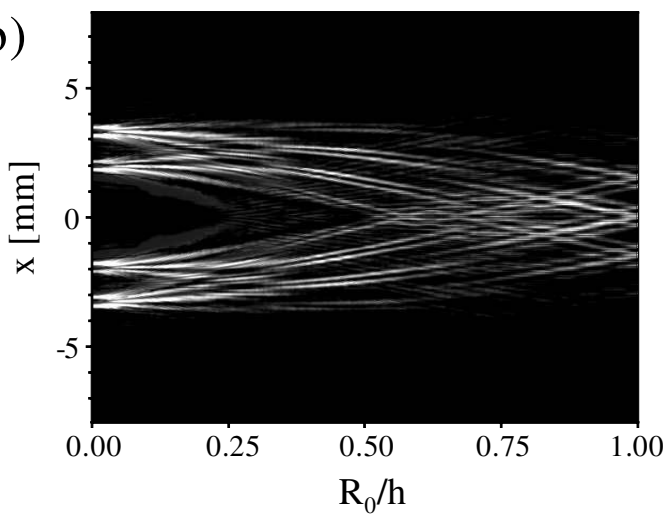

Figure 2. Output 2D display of all diffration patterns generated by a Cantor grating (level 4): (a) numerically obtained; (b) experimentally obtained.

From the experimental chart, the values of the irradiance at several particular transverse planes were obtained, and from them, the degree of self-similarity of the intensity distributions in the whole Fresnel region was computed. This characteristic function was calculated as the normalized correlation between an intensity profile of a given Fresnel diffraction pattern of the input function and a magnified version of it, by means of the following equation [4]:

$$
C(m)=\frac{\int(f(x)-\bar{f})\left(f(x / m)-\bar{f}_{m}\right) \mathrm{d} x}{\left[\int(f(x)-\bar{f})^{2} \mathrm{~d} x \int\left(f(x / m)-\bar{f}_{m}\right)^{2} \mathrm{~d} x\right]^{1 / 2}}
$$

where $\bar{f}$ and $\bar{f}_{m}$ are the mean values of Fresnel patterns $f(x)$ and $f(x / m)$ respectively, and $m$ is the magnification factor. If the Fresnel pattern $f(x)$ has an exact fractal property, $f(x / m)$ is identical to $f(x)$ and then from equation $(10) C(m)=1$. In contrast, if $C(m)$ does not take a value close to unity, the corresponding Fresnel pattern may be said to be non self-similar. In this way, the degree of self-similarity is expected to be quantitatively expressed by $C(m)$.

Figure 3 shows the evolution of the self-similarity degree in free-space propagation obtained from the experimental intensity distributions of figure 
2(a), for the scale factor $m=4$. From this plot it can be noted that the selfsimilarity varies depending upon the propagating distance. The function $C(m)$ starts with its maximun value at the object plane, as expected, but falls rapidly showing poor self-similarity very close to the aperture. From $R_{0} / h=0.5$ the local mean value of this function grows as the observation plane moves towards the far field.

\section{Conclusions}

A simple experimental setup is proposed to obtain a simultaneous display of all diffraction patterns for $1 \mathrm{D}$ fractal structures. The design was based on the fact that the free-space propagation of a converging wavefront illuminating an input transparency provides all the diffraction patterns of this signal continuously distributed along a finite segment of the optical axis. The key optical element in the proposed setup is a varifocal lens which is used as a selective image-forming element to obtain the images of these diffraction patterns but superimposed in a single 2D display. The optical setup uses conventional optical refractive elements plus a commercially available ophthalmic varifocal lens. Every pattern in the display, which is characterized by its propagation distance, can be identified and its transversal magnification calculated through a simple formula. It is shown that this

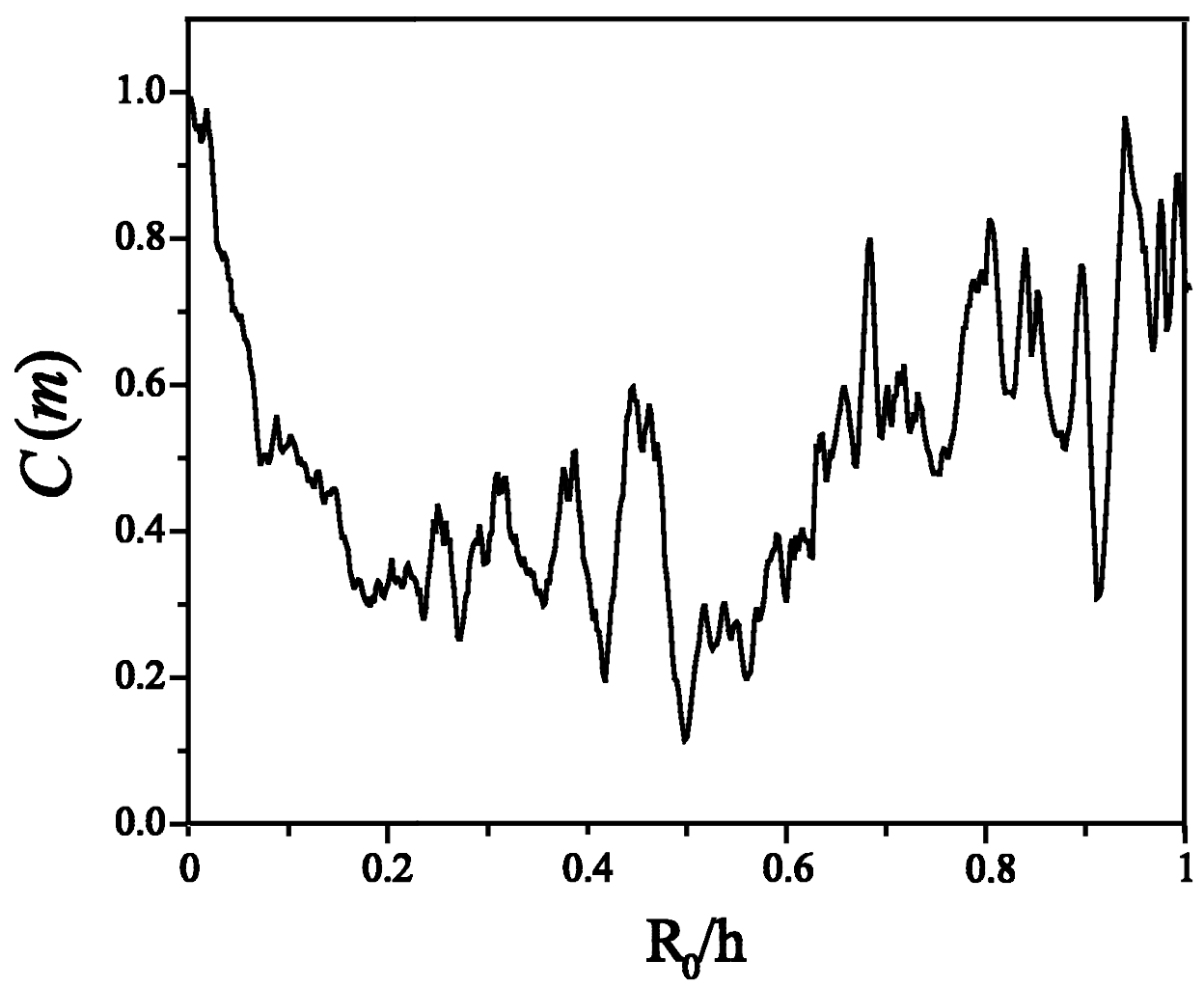

Figure 3. Evolution of the self-similarity of the diffracted intensity obtained from the experimental result of figure $2(b)$. 
kind of experimental data can be successfully used to calculate some relevant parameters of fractal structures such as the self-similarity. Continuous monitoring of the diffraction pattern under propagation could also be useful to perform other tasks, for example, to detect the presence of singularities or noise located upon a fractal structure. Exploring further applications of this type of experimental display in this and other areas remains a rich direction for further research. In particular, one very useful application of the method would be to investigate the fractal Talbot images [8]; i.e. the fractal structure of the diffracted field produced by a binary (not fractal) grating. The proposed experimental setup can be used to give a synoptic image of the diffraction patterns in the Talbot cell behind the grating, where the wave has a rich fractal structure in transverse, longitudinal and even in certain diagonal planes.

\section{Acknowledgments}

This work was supported by the project GV99-100-1-01 of the Conselleria de Cultura, Educació i Ciència. Generalitat Valenciana, Spain. The ophthalmic varifocal lens used in the experiments was kindly provided by INDO, S.A. O. Trabocchi and S. Granieri acknowledge partial support from the Consejo Nacional de Investigaciones Científicas y Técnicas CONICET (Argentina) and Universidad Nacional de La Plata. O. Trabocchi also received support from the postgraduate fellowship programme of the Universitat de Valencia. The authors would like to thank Dr Genaro Saavedra and the referees of this paper for their valuable comments and suggestions.

\section{References}

[1] Mandelbrot, B., 1977, Fractal Geometry of Nature (New York: Freeman).

[2] Allain, C., and Cloitre, M., 1986, Phys. Rev. B, 33, 3566.

[3] Uozumi, J., Kimura, H., and Asakura, T., 1990, J. Mod. Opt., 37, 1011. Uozumi, J., Kimura, H., and Asakura, T., 1991, J. Mod. Opt., 38, 1335.

[4] Sakurada, Y., Uozumi, J., and Asakura, T., 1992, Pure Appl. Opt., 1, 29.

[5] Alieva, T., and Agulló-López, 1996, Opt. Commun., 125, 267.

[6] Gaskill, J. D., 1978, Linear Systems, Fourier Transforms and Optics (New York: Wiley), Chapter 10.

[7] Jalie, M., 1984, The Principles of Ophthalmic Lenses (London: The Association of Dispensing Opticians), pp. 362-3.

[8] Berry, M.V., and Klein, S., 1996, J. Mod. Opt., 43, 2139. 